\section{How can multi-professional education support better stewardship?}

\author{
Nuno Rocha Pereira, ${ }^{1-3}$ \\ Enrique Castro-Sanchez, ${ }^{4}$ \\ Dilip Nathwani ${ }^{5}$
}

${ }^{1}$ Infectious Diseases Department, Centro

Hospitalar São João, Porto, Portugal; ${ }^{2}$ Infection and Antimicrobial Resistance Prevention and Control Unit, Centro Hospitalar São João, Porto, Portugal; ${ }^{3}$ Faculty of Medicine Porto University, Porto, Portugal; ${ }^{4}$ NIHR Health

Protection Research Unit, Healthcare Associated Infection and Antimicrobial Resistance, Imperial College, London, UK; ${ }^{5}$ Ninewells Hospital and Medical School, Dundee, UK

\begin{abstract}
Antimicrobial stewardship is widely accepted as an efficient strategy to combat the growing threat of antimicrobial resistance. Education is one of the cornerstones of successful antimicrobial stewardship programs. There is also general agreement that antimicrobial stewardship is a team effort that must involve the whole continuum of healthcare workers. Providing adequate education for all different professionals although challenging is deemed crucial to achieve good results. This paper reviews the different strategies available to educate the multiple healthcare workers, discusses how education can improve antimicrobial stewardship programs and outlines some of the challenges faced and research gaps that need to be addressed in order to improve education in this field.
\end{abstract}

\section{Introduction}

Antimicrobial resistance is a major medical problem and in recent years we have witnessed a dramatic increase in resistance with important consequences in terms of morbidity and mortality. ${ }^{1}$ It is also widely known that emergence and spread of resistant strains is strongly linked with overuse or inappropriate use of antimicrobials. ${ }^{2}$ Additionally, the investigation of new antimicrobial agents with the ability to overcome resistance mechanisms is at a crossroad. $^{3}$ Therefore antimicrobial stewardship will be increasingly important as a set of tools to enhance and monitor the rational use of antibiotics, and by doing so accomplishing a reduction in antimicrobial resistance and preserving antimicrobials for future generations.

In contrast with other drugs, antimicrobials are widely used and prescribed by large range of health professionals, with some of them lacking in knowledge about important aspects of antimicrobial stewardship. There is also evidence that behaviour around prescribing and management of antimicrobials is strongly affected by social interactions between the different professions providing healthcare. ${ }^{4}$ On the other hand strong and often erroneous beliefs about antibiotics are common in patients and citizens. All the reasons aforementioned provide the rationale supporting why every healthcare professional in contact with patients should learn at least basic principles of antimicrobial stewardship and antimicrobial resistance. Indeed, the link between educated health care professionals and good care and patient outcomes has long been recognised. For example, in the mid-1800s, Florence Nightingale ${ }^{5}$ campaigned that good nursing care saved lives, and good nursing care depended on educated nurses. The first nursing education programme began in London in 1859 soon spreading quickly in the UK, the USA, Germany, and Scandinavian countries. ${ }^{6}$ Despite this and much evidence to support this across the various healthcare professional domains the gap between health delivery and provision of high quality, resourced and sustained education provision so as to have an educated and competent workforce remains.

The 21 st century is challenging environment for healthcare professionals due to the complex and costly care. The challenge of antimicrobial resistance leading to drug resistant infections is a salient example of this. Alongside the rapid pace of change in health, there is a parallel revolution in education. The explosive increase not only in total volume of information but also in ease of access to it. Professional education has not kept pace with these challenges for many reasons that include poor leadership to improve health systems, professional tribalism, a mismatch of competencies to patient and population needs; poor teamwork, persistent gender stratification of professional status; narrow technical focus without broader contextual understanding, episodic encounters rather than continuous care, a predominant hospital orientation at the expense of primary care, quantitative and qualitative imbalances in the professional labour market; and weak leadership to improve health-system performance.

To address these challenges a major design of the health professional education has been recommended. ${ }^{7}$ This requires a
Correspondence: Nuno Miguel Rocha Pereira, Serviço de Doenças Infecciosas, Centro Hospitalar São João, Alameda Prof. Hernâni Monteiro, 4200 Porto, Portugal.

Tel.: +35.1964280481

E-mail: nunopereira85@gmail.com

Key words: Antimicrobial stewardship, education; antimicrobials; prescribers.

Contributions: NRP and ECS reviewed the literature and wrote the paper; DN participated in the definition of paper structure, reviewed the paper and made suggestions to the final version.

Conflict of interest: the authors declare no potential conflict of interest.

Acknowledgements: this work was supported by the National Institute for Health Research Health Protection Research Unit (NIHR HPRU) [HPRU-2012-10047] in Healthcare Associated Infection and Antimicrobial Resistance at Imperial College London in partnership with Public Health England. The views expressed are those of the author(s) and not necessarily those of the National Health Service, the NIHR, the Department of Health or Public Health England. The content of this article is solely the responsibility of the authors and any conclusions or interpretations may not reflect the funders' views.

Received for publication: 4 October 2016

Revision received: 24 January 2017.

Accepted for publication: 27 January 2017.

This work is licensed under a Creative Commons Attribution-NonCommercial 4.0 International License (CC BY-NC 4.0).

(C) Copyright N.R. Pereira et al., 2017

Licensee PAGEPress, Italy

Infectious Disease Reports 2017; 9:6917

doi:10.4081/idr.2017.6917

global, systems and multi-professional approach. The delivery of infection treatment and antimicrobial stewardship is truly multi-professional.

Despite education is widely accepted as a fundamental tool to tackle resistance clear gaps still exist both in undergraduate curricula and lifelong learning. For example the inconsistency of teaching on clinical infectious diseases in undergraduate medical curricula and the perceived unpreparedness for practice around antimicrobial prescribing ${ }^{8,9}$ has led to calls to address undergraduate teaching urgently. ${ }^{10,11}$

However, education of clinicians and other healthcare professionals is needed, delivery of such education to a wide spectrum of different professionals is challenging because of different backgrounds, 
beliefs and cultural factors. Traditionally education about antimicrobial stewardship has been part of the competences of a multidisciplinary antibiotic team ${ }^{8}$ but this strategy might be insufficient in addressing a wide variety of health professionals. This review discusses the challenges in educating the different healthcare providers and how efforts around education can boost antimicrobial stewardship across the continuum of healthcare.

\section{Education of clinicians}

Antimicrobials are unique drugs because they do not target directly the patient but instead affect the growth, replication and whole ecology of both invading pathogens and the microbiome. ${ }^{10,12}$ By doing so antimicrobials can have impact not only in the patient being treated but also for others who may become colonized or infected with resistant bacteria. ${ }^{12}$ Despite the aforementioned complexity inherent to the use of antimicrobials, all clinicians are potential antimicrobial prescribers ${ }^{13,14}$ and it is likely that the high level of inappropriate use of antimicrobials is largely a result of a misbalance between the high level of skills required for appropriate use and the low level of training offered to most clinicians. ${ }^{13}$ Although medical microbiology and infectious diseases specialties are specifically trained to use antimicrobials and treat infections, many infections are secondary to other medical conditions and prescription of antimicrobials is a responsibility of the team taking care of the patient. ${ }^{15}$ Recognizing this fact means that all potential prescriber clinician should receive adequate education in antimicrobial stewardship. ${ }^{11,15}$

Albeit the wide recognition by medical societies and governmental agencies that education around infection management and correct antimicrobials use is fundamental for any clinician, important knowledge gaps are still identifiable both in undergraduate medical students and practising clinicians. A study by Abbo et al. ${ }^{8}$ in American students showed that only $40 \%$ of students were familiar with the term antimicrobial stewardship and less than one third understood the correct spectrum for different antimicrobials. The participants in this study also had a suboptimal performance on questions regarding mechanisms of antimicrobial resistance, recognition of C. difficile infection and management of urinary tract infections. Another study conducted in Europe showed that students were least confident in choosing combination therapies, correct dose, interval of administration and duration of antibiotics and in making the decision to not prescribe in cases of diagnostic uncertainty. ${ }^{9}$ In both studies students recognised that more education on antimicrobial use is necessary. Also a recent systematic review of 57 studies $^{16}$ showed that less than $25 \%$ of clinicians knew the correct prevalence of resistance and that more than $75 \%$ believed that too short antibiotic duration contributed to resistance. Interestingly, this review also showed a process of diffusion of responsibility with a considerable number of clinicians attributing blame for antibiotic resistance to other healthcare settings. These gaps have been recognized for some time by medical professional societies and public health societies, ${ }^{17}$ and calls into action have been issued. Medical schools are probably one of the most important players in this field because of the exceptional opportunity they have to shape behaviour of future clinicians. ${ }^{10}$ However information about the content and quality of teaching in this area is lacking. ${ }^{17}$ Some countries have already put into practice the definition of minimum contents to be incorporated in medical schools curricula but how this regulations are being put in practice and the results achieved are still unknown and matter for further debate.

Undergraduate medical education is only a piece of a wider complex puzzle and it is easily understandable that education of practising clinicians is of outmost importance to achieve better antimicrobials prescribing practices. Behavioural sciences clearly demonstrate that changing behaviour is a lot harder than shaping it ${ }^{10}$ and is also largely known that once medical doctors become qualified and gain experience, then their views and behaviours become established and are very hard to change. ${ }^{11}$ Despite all these difficulties there are several possible strategies with proven results that can be used to educate clinicians. The review by Lee et al. ${ }^{11}$ describes the following possible education interventions in the setting of antimicrobial stewardship: interactive seminars; mailing campaigns; small-group education focusing on evidence based medicine and communication skills; educational outreach visits; guidelines and leaflets; combination of several strategies.

The evidence presented reports an average reduction in antibiotic prescription of $39.1 \%$ and a reduction in antibiotic inappropriate antibiotic prescriptions of $41 \%$. Despite the difficulty to compare available data to define which educational interventions show better outcomes, it seems that small group education and production of guidelines and leaflets are the ones that have greater effects. Other educational tools based on E-learning are emerging and can fulfil some of the gaps of more traditional methods of education, however measurement of the effectiveness of these approaches in changing and improving antimicrobial prescribing is still needed. ${ }^{18}$

Although strong evidence of efficacy of educational programs about antimicrobial stewardship exists it is controversial that this kind of interventions alone can achieve good results. Indeed a very recent document issued by the Infectious Diseases Society of America ${ }^{14}$ recommends against stewardship programs solely based on educational interventions and although these interventions have more acceptance among clinicians it is generally recognized that they need to be put in place in a context of a strong antimicrobial stewardship team that also conducts other types of restrictive and supportive interventions. ${ }^{11}$ Nonetheless changing clinicians behaviour around antimicrobial prescriptions is challenging and contextual, cultural and behavioural factors must be addressed when planning educational interventions. ${ }^{19}$ The absence of guidelines, lack of diagnostic resources or workforce, patient characteristics and expectations, all must be considered when designing such interventions. Also cultural factors must be addressed and it is known that there are a set of unwritten but widely accepted cultural rules around prescribing, the so called prescribing etiquette. ${ }^{15}$ These set of rules are very often based more on the popular sports adagio do not switch a winning team rather than in good quality evidence. Recognizing the existence of these cultural beliefs is crucial to produce effective educational contents. One possible strategy is to involve opinion leaders within clinical specialties in the development and adoption of local guidelines and evidence from a few studies indicate that peers and opinion leaders perceptions and behaviours have greater influence on prescribing when compared to local policies. ${ }^{17}$ The last set of factors to be considered are behavioural and include things like lack of awareness or agreement with guidelines, fear of withholding or adjusting antimicrobials, resistance to change practice and perceived loss of autonomy. ${ }^{19}$ To address this factors principles imported from social sciences may need to be used.

Another special consideration to be made is that antimicrobial resistance is not a hospital problem and most of the antimicrobial prescriptions are done in community setting. However most of the efforts and financing of antimicrobial stewardship programs have been put in the hospitals and prescribers in community are often forgotten when designing educational interven- 
tions. ${ }^{11}$ Consequently, few data exist about interventions in ambulatory medicine. Nonetheless a randomized clinical trial by Gerber et al. ${ }^{20}$ gives encouraging evidence of efficacy of such interventions in ambulatory care.

The final objective of any antimicrobial stewardship intervention, whether performed in outpatient or inpatient setting, is to change and improve clinicians antimicrobial prescribing behaviour. ${ }^{17}$ Traditional measures of education efficacy such as increased knowledge about antimicrobial stewardship principles are insufficient to evaluate success of this kind of intervention because increased knowledge is not directly related to change in prescribing behaviour. Acknowledging this should direct educational interventions toward more interactive or dynamic techniques such as education associated with specific episodes of patient care or combined with audit and feedback, which are more prone to influence prescribing behaviour. ${ }^{17}$ Education of clinicians is a cornerstone in any antimicrobial stewardship program and when performed in addition to other strategies it can certainly change clinicians use of antimicrobials and also can augment acceptance and awareness for the antimicrobial stewardship team interventions, reaching the global goal of better prescribing of antimicrobials.

\section{Education for other healthcare professionals}

Multi-professional education and training have been highlighted as key areas for action to facilitate optimal clinical practice ${ }^{21}$ with significant resources and investment already allocated. Further, there is a growing interest in ensuring that such education is coordinated and comprehensive, ${ }^{22}$ following clinicians from undergraduate to the diversity of early and late professional careers stages.

\section{Nurses and infection control/preven- tion practitioners}

Institutional antimicrobial stewardship improvement programmes have traditionally designated physicians and pharmacists with advanced training in infectious diseases as core staff, with the participation of other healthcare professionals suggested as beneficial but considered as optional. ${ }^{23}$ More recent national ${ }^{24}$ and international ${ }^{14}$ recommendations and policies have emphasised a need for collaborative and integrated approaches. The framing of antimicrobial stewardship together with infection prevention and control within the patient safety agenda perspective ${ }^{25}$ may have contributed to the gradual incorporation of healthcare professionals such as nurses onto stewardship interventions. ${ }^{26,27}$

As the contribution of nurses within antimicrobial stewardship interventions has gained recognition, increased attention has been given to their education and training related to this area, reflecting perhaps the knowledge deficits and theory-practice gaps already reported by medical students ${ }^{9}$ and trainee doctors ${ }^{28}$ as well as in other disciplines. ${ }^{29,30}$ The initial experiences published already suggest that increased education in stewardship for nurses can have benefits for healthcare organisations. ${ }^{31,32}$ In healthcare systems with infection control/prevention practitioners next to nursing staff the topics discussed would also apply to this group of professionals.

If antimicrobial quality improvement programmes in acute care can be optimised by educating nurses and supporting their involvement, the impact can be even more sizable on nursing homes and long-term care institutions, in view of results already obtained. $^{33}$ Interestingly, an increasing number of authors have already proposed the expansion of the scope of nursing participation in stewardship, leaving behind a focus on tasks related to the administration of antimicrobials, the collection of microbiological samples or as mere reminders to encourage prescribers to comply with guidelines and policies, and endorsing their involvement in the design, implementation and evaluation of antimicrobial stewardship programmes. ${ }^{34}$ The impact of these additional competences on current and future educational needs and demands is yet to be fully appreciated.Finally, independent nurse prescribers $^{35}$ have emerged not just in the UK but also worldwide. ${ }^{36}$ Early reports evaluating their impact point towards a beneficial influence on antimicrobial use at community level. ${ }^{37}$ This will likely increase demand for the development of relevant educational opportunities focused on clinical decision-making and concepts of stew$\operatorname{ardship.~}^{38}$

\section{Pharmacists}

The growth and progression of pharmacists specialized in infectious diseases since the early 2000s has naturally facilitated their leadership role within antimicrobial stewardship interventions. The range of activities conducted by pharmacists is ample, from expert clinical consultation and advice, ${ }^{39}$ to coordination of antimicrobial stewardship committees and guidelines ${ }^{40}$ and even the development of antimicrobial advice smartphone apps. ${ }^{41}$
In the UK, some early educational approaches specifically directed to pharmacists with a holistic perspective about antimicrobial stewardship included the MSc Infection at Imperial College London in 2012. The role variety was later reflected in the expert professional curriculum for antimicrobial pharmacists developed by the Royal Pharmaceutical Society in 2013, a competency-based programme across five core areas that include clinical, educational, policy-making and leadership domains. ${ }^{42}$

Achieving proficiency in the areas included on the curriculum may have been challenging for pharmacists in some settings, due to the focus on educational outcomes related to infectious diseases given by national pharmacy regulatory and education bodies and the lack of a clear remit for antimicrobial stewardship. Some experiences have attempted to ease the transition from undergraduate to professional stages and increase the awareness and knowledge about infectious diseases and stewardship, using simple methodologies such as active learning strategies (i.e., journal clubs and mini-lectures) resulting in greater understanding of stewardship initiatives for students. ${ }^{43}$

\section{Other healthcare workers}

The global impact of antimicrobial resistance on human and animal health demands the involvement in stewardship efforts of a larger cadre of professionals. ${ }^{7}$ For example, public health agencies are ideally placed to advocate for such efforts across care settings and levels. The essential contribution of other professional groups such as veterinary doctors and dentists is slowly receiving attention and therefore the educational opportunities related to both groups is also under evaluation. Dentists prescribe $\sim 10 \%$ of antibiotics dispensed in UK community pharmacies,${ }^{44}$ and although at least one person a month dies in the UK due to an acute dental infection, ${ }^{45}$ education efforts may be primarily directed to enhance communication and health promotion skills to reduce requests for antibiotics from patients complaining of toothaches. ${ }^{46}$ In line with prescribing by physicians, interventions for dentists based on audits and feedback have resulted in improved antimicrobial prescribing. ${ }^{47}$ It is encouraging to note that in the recent revised competency framework for prescribing by dentists ${ }^{48}$ the understanding of antimicrobial resistance and the roles of infection prevention, control and antimicrobial stewardship measures have been specified, therefore signaling the recognition by all healthcare professions of the importance achieved by quality 
antiviviu provitivins anu siorraruonip. Types of educational activities and minimal contents to deliver

A variety of pedagogies have been used in different studies regarding educational approaches to teach antimicrobial stewardship. For example, a nationwide evaluation of antimicrobial stewardship content in UK undergraduate courses in medicine, pharmacy, nursing, veterinary medicine and dentistry identified the use of several teaching methodologies including primarily lectures but also case presentations by the students and group discussions that followed a problem-based learning structure. ${ }^{4}$ The evaluation approaches ranged from multiple choice questions to long essays. Interestingly, one of the participating universities lacked any kind of evaluation. Additionally, a minority of universities taught students of different disciplines together.

\section{E-learning tools}

There has been increasing appreciation of the role of e-learning in stewardship. ${ }^{18} \mathrm{E}$ learning and online platforms are being used to deliver antimicrobial stewardship education. Forward-thinking examples include initiatives such as the online educational package developed by the Scottish Antimicrobial Prescribing Group (SAPG), which included training materials for undergraduate and postgraduate healthcare workers. Again in Scotland, the Doctors Online Training System, a web-based education resource for all trainee doctors, pharmacists and non-medical prescribers also includes education about prudent antibiotic use. ${ }^{49} \mathrm{At}$ a global scale, massive open online courses (MOOC's) have now been used to reach large numbers of interested clinicians, although their impact and sustainability are yet to be evaluated. Other online platforms used to train and educate clinicians have included serious electronic games allowing prescribers to practice the diagnosis and management of a series of virtual cases, ${ }^{50}$ and novel immersive virtual reality environments designed to evaluate the responses of family doctors to patients requesting antibiotics. $^{51}$

\section{Written local guidelines and leaflet/posters/outdoor campaigns}

Written guidelines have become the mainstay of supportive and educational measures for prescribers. ${ }^{52}$ For example, a survey of specialist antimicrobial pharmacists in National Health Service Organisations in England in $2013^{53}$ identified how virtually all respondents reported having policies or guidance in place, depending on the clinical area. The adoption of a classical multifaceted educational and organisational approach that includes the use of guidelines adapted to local susceptibility data, dissemination through handouts and posters combined with educational sessions and clinical pharmacist leadership has achieved enhanced adoption and use of guidance and significantly better antimicrobial prescribing, even in relative low resource settings. ${ }^{54}$ This multi-faceted, workplace based approach has much to commend it. As a note of caution, the implementation of guidelines in clinical practice should not dismiss the effect that social interactions can have on their ultimate use and efficacy. ${ }^{55}$

\section{Daily ward rounds: face-to-face edu- cation}

Academic and professional detailing, including the attendance of pharmacists during ward rounds and follow up face to face interactions appraising the performance of prescribers have been useful to improve the overall quality and reduce the volume of antimicrobial prescriptions. ${ }^{13,56-58}$ These interactions provide point-of-care, contextual advice that can be seen as less aggressive and rigid than simply matching clinical decision-making to existing guidelines, which can be dismissed by prescribers. ${ }^{59}$ The provision of formalised antimicrobial education during employment but outside clinical practices, using short briefs, seminars and workshops is routinely reported. A study ${ }^{53}$ surveying hospitals nationwide exploring the delivery of antimicrobial education concluded that nearly $2 / 3$ of hospitals offered antimicrobial training to pharmacists and doctors at induction, with regular post-induction refresher sessions for these groups. Of concern, the percentage of organisation that included nursing staff on these sessions was less frequent. Workplace based training and education may offer an excellent opportunity to address practical knowledge deficits from undergraduate education. For instance, a recent study highlighted the limited education received by medical trainees about antibiograms, ${ }^{60}$ where more than half of surveyed participants had not received any education on this topic and with a third failing to accurately answer the more challenging questions.

\section{The Participatory Action Research methodology applied to AS}

Issues of engagement with optimal antimicrobial behaviours seem to be frequent regardless of the pedagogy used to deliver antimicrobial education. For such reason, innovative methodological approaches such as participatory action research (PAR) have been suggested as a valuable adjunct within traditional multimodal interventions ${ }^{19}$. The DUMAS and IMPACT studies systematically analysed the variety of antimicrobial prescribing determinants at a hospital and community setting with the aim to optimise the effectiveness of the interventions available. ${ }^{19}$ As unique contribution of the PAR methodology, the multidisciplinary team was engaged and motivated to actively lead the design and changing of their practice, addressing intervention implementation challenges. Regardless of the interventions finally selected by the participants, the iterative process of reflecting on action, learning from each other and gradually building the different components and steps of the programme appears particularly apt in view of the collaborative and problem-solving focus of clinical practice.

Research and improvement methodologies that strive to understand the clinical, behavioural and structural determinants of antimicrobial prescribing highlight the need to design specific interventions for different professional groups. Whether these interventions are based on behaviours identified, ${ }^{61,62}$ different communication styles, ${ }^{63}$ or even approaches to uncertainty or asymmetry of clinical information, ${ }^{64}$ there may be benefit for educational activities in considering these characteristics to enhance their impact, perhaps even echoing experiences profiling the personality of different healthcare workers to guide change strategies. ${ }^{65}$

Whilst the need to improve antimicrobial prescribing knowledge, attitudes and behaviours at crucial formative stages is well accepted, a consensus on the minimal essential content that antimicrobial stewardship curricula should include may need further debate. Whilst antimicrobial stewardship is a concrete, well-defined bundle of behaviours and actions, ${ }^{66}$ the multiple professional groups involved and the behavioural, cognitive and relational skills required for optimal prescribing may not facilitate arriving at a core content of what should be taught. ${ }^{67}$ Even if such agreement could be reached, mirroring for instance the expert professional curriculum for pharmacists previously mentioned, it may be difficult to determine the optimal duration of each module and the level of necessary time investment, in view of the lack of benchmarks. Once again, the disparity of time commitment apportioned to antimicrobial stewardship by UK universities ${ }^{4}$ suggests that this area demands further exploration.

The development of competency frameworks may also be affected by the difficulty 
in neatly describing and packaging those skills such as communication and assertiveness, increasingly recognised as playing a crucial role in the negotiating and decisionmaking that surround antibiotic prescribing. ${ }^{68}$ Furthermore, as antimicrobial decisions are carried out within increasingly multiprofessional and in complex landscapes where clinical, financial and structural competing demands coexist, training frameworks would benefit from fostering skills that are not simply technical (i.e., knowledge about antibiotics) but include also non-technical skills. ${ }^{7}$

Finally, whilst the introduction of decision support tools increasingly based on artificial intelligence or complex algorithms may have beneficial effects on individual patients, it may also lead to potential drawbacks for antimicrobial prescribers, including overreliance on software that could end up in deskilling, as well as perceptions of inflexibility that may result in frustration. ${ }^{69,70}$

\section{Education metrics: process, structural and outcomes meas- urement}

Associated to the challenge of identifying the appropriate content and optimal duration of antimicrobial stewardship educational interventions, the selection of metrics that faithfully reflect the impact of such interventions also requires thought.

\section{Ratio of engaged professionals}

For example, and drawing from the indicators used in infection prevention and control or hand hygiene programmes, ${ }^{71}$ the number of professionals engaged in antimicrobial stewardship may reflect improvements or shifts towards optimal institutional climates focused on appropriate use of antimicrobials. Similarly, and in view of the proposed composition of stewardship teams, it may be possible to suggest optimal professional ratios; however, the comparability of these ratios on different organisations will clearly depend on the roles carried out by each professional group.

\section{Quality of prescription indicators}

Ideally, antimicrobial education programs should consider the impact of different education interventions on the variety of antimicrobial prescription quality indicators available and that may be relevant or suitable to each local organisation. For example, whilst an emphasis has been placed on volumes of prescribed antimicrobials ${ }^{72}$ and such indicator is clearly essential, the appropriateness of all decisions along the clinical management path may be reflected on the education support to be provided. ${ }^{73}$ For example, appropriate diagnosing would lead to fewer antibiotics used empirically, or at all. ${ }^{74}$ However, clinicians such as nurses may need to be supported in their consideration of the importance and quality and timeliness of biological sample collection, and physicians may require additional training to understand the outputs provided by new screening and diagnostics tests. ${ }^{75}$ Such added support may be even more crucial considering the current impetus to develop and deploy rapid diagnostic tests. Inappropriate or redundant microbial coverage may require education about the evidence available on local epidemiological patterns. ${ }^{76}$

\section{Conclusions}

In conclusion we refer back to the premise of this paper- how can multi-professional education support better stewardship? Indeed, the great physician William Osler recognized that the first duties of the physician is to educate the masses not to take medicine. Perhaps we could extend this to the infection communities first duty is to educate all clinicians only to prescribe antibiotics only when necessary and what necessary means. To support this we suggest the following key messages: i) stewardship education requires a re-design of the health professional education system within an organization and a multi-professional and team based approach; ii) identifying the unmet education needs to the professional involved in prescribing, its delivery and evaluation and recognizing the context of where the care is provided is a core step. Simple messages, relevant to the professional and clinical context are core to success of the interventions; iii) developing competencies that are relevant and appropriate for the above are a fundamental step before identifying a range of effective active educational solutions or interventions, based on evidence, that will allow not only support knowledge transfer but also retention of knowledge that is sustainable and leads to practice change. This needs to be a continuous process, often supported in the work place and where possible developed and delivered as a team; iv) measuring engagement with educational activity should be a core requirement of the educational process whereas how best to measure impact on prescribing is perhaps a more challenging but worthwhile objective.

\section{References}

1. Dellit TH, Owens RC, McGowan JE, Jr., et al. Infectious Diseases Society of America and the Society for Healthcare Epidemiology of America guidelines for developing an institutional program to enhance antimicrobial stewardship. Clin Infect Dis 2007;44:159-77.

2. Goossens H. Antibiotic consumption and link to resistance. Clin Microbiol Infect 2009;15:12-5.

3. Nathan C, Cars O. Antibiotic resistance-problems, progress, and prospects. New Engl J Med 2014;371: 1761-3.

4. Castro-Sanchez E, Drumright LN, Gharbi M, et al. Mapping antimicrobial stewardship in undergraduate medical, dental, pharmacy, nursing and veterinary education in the United Kingdom. PLoS One 2016;11:e0150056.

5. F N. Notes on Nursing. What it is, and what it is not. 1st ed. New York: Apleton and Company; 1860.

6. M B. Florence Nightingale: the making of an icon. New York: Farrar, Straus and Giroux; 2008.

7. Frenk J, Chen L, Bhutta ZA, et al. Health professionals for a new century: transforming education to strengthen health systems in an interdependent world. Lancet 2010;376:1923-58.

8. Abbo LM, Cosgrove SE, Pottinger PS, et al. Medical students' perceptions and knowledge about antimicrobial stewardship: how are we educating our future prescribers? Clin Infect Dis 2013;57:631-8.

9. Dyar OJ, Pulcini C, Howard P, et al. European medical students: a first multicentre study of knowledge, attitudes and perceptions of antibiotic prescribing and antibiotic resistance. J Antimicrob Chemother 2014;69:842-6.

10. Pulcini C, Gyssens IC. How to educate prescribers in antimicrobial stewardship practices. Virulence 2013;4:192-202.

11. Lee CR, Lee JH, Kang LW, et al. Educational effectiveness, target, and content for prudent antibiotic use. Biomed Res Int 2015;2015:214021.

12. Ashiru-Oredope D, Cookson B, Fry C, Advisory Committee on Antimicrobial $\mathrm{R}$, Healthcare Associated Infection Professional Education S. Developing the first national antimicrobial prescribing and stewardship competences. J Antimicrob Chemother 2014;69:28868.

13. Cisneros JM, Neth O, Gil-Navarro MV, et al. Global impact of an educational antimicrobial stewardship programme on prescribing practice in a tertiary hos- 
pital centre. Clin Microbiol Infect 2014;20:82-8.

14. Barlam TF, Cosgrove SE, Abbo LM, et al. Executive summary: implementing an antibiotic stewardship program: guidelines by the Infectious Diseases Society of America and the Society for Healthcare Epidemiology of America. Clin Infect Dis 2016;62:1197-202.

15. Charani E, Castro-Sanchez E, Holmes A. The role of behavior change in antimicrobial stewardship. Infect Dis Clin North Am 2014;28:169-75.

16. McCullough AR, Rathbone J, Parekh S, et al. Not in my backyard: a systematic review of clinicians' knowledge and beliefs about antibiotic resistance. J Antimicrob Chemother 2015;70:246573.

17. Ohl CA, Luther VP. Health care provider education as a tool to enhance antibiotic stewardship practices. Infect Dis Clin North Am 2014;28:177-93.

18. Rocha-Pereira N, Lafferty N, Nathwani D. Educating healthcare professionals in antimicrobial stewardship: can online-learning solutions help? J Antimicrob Chemother 2015;70:31757.

19. van Buul LW, Sikkens JJ, van Agtmael MA, et al. Participatory action research in antimicrobial stewardship: a novel approach to improving antimicrobial prescribing in hospitals and long-term care facilities. J Antimicrob Chemother 2014;69:1734-41.

20. Gerber JS, Prasad PA, Fiks AG, et al. Effect of an outpatient antimicrobial stewardship intervention on broadspectrum antibiotic prescribing by primary care pediatricians: a randomized trial. JAMA 2013;309:2345-52.

21. Department of Health UK. Five year antimicrobial resistance strategy 2013 to 2018. 2016. Available from: https://www.gov.uk/government/upload s/system/uploads/attachment_data/file/ 244058/20130902_UK 5 year AMR strategy.pdf. Accessed 24/09/2016

22. Shekarchian S, Schwartz BS, Teherani A, et al. Is it time for a coordinated and longitudinal approach to antibiotic stewardship education? Clin Infect Dis 2016;63:848-9.

23. Olans RN, Olans RD, DeMaria A, Jr. The critical role of the staff nurse in antimicrobial stewardship - unrecognized, but already there. Clin Infect Dis 2016;62:84-9.

24. Nathwani D, Sneddon J, Patton A, Malcolm W. Antimicrobial stewardship in Scotland: impact of a national programme. Antimicrob Resist Infect Control 2012;1:7.
25. Carlet J. World alliance against antibiotic resistance: The WAAAR declaration against antibiotic resistance. Medicina intensiva / Sociedad Espanola de Medicina Intensiva y Unidades Coronarias 2015;39:34-9.

26. Gillespie E, Rodrigues A, Wright L, et al. Improving antibiotic stewardship by involving nurses. Am J Infect Control 2013;41:365-7.

27. Charani E, Cooke J, Holmes A. Antibiotic stewardship programmeswhat's missing? J Antimicrob Chemother 2010;65:2275-7.

28. Navarro-San Francisco C, Del Toro $\mathrm{MD}$, Cobo J, et al. Knowledge and perceptions of junior and senior Spanish resident doctors about antibiotic use and resistance: results of a multicenter survey. Enferm Infecc Microbiol Clin 2013;31:199-204.

29. Berr L, Donaldson N, Hatzipanagos S, et al. The impact on dental students' knowledge in three European countries through an online module on antibiotic prescribing: a preliminary study. Bull Group Int Rech Sci Stomatol Odontol 2013;51:e25-6.

30. Speksnijder DC, Jaarsma DA, Verheij TJ, Wagenaar JA. Attitudes and perceptions of Dutch veterinarians on their role in the reduction of antimicrobial use in farm animals. Prev Vet Med 2015;121:365-73.

31. Weddle G, Goldman J, Myers A, Newland J. Impact of an educational intervention to improve antibiotic prescribing for nurse practitioners in a pediatric urgent care center. J Pediatr Health Care 2016:S0891-5245.

32. Nutall SE DC, Mills R. Evaluation of the antibiotic prescribing of nurse practitioners trained to prescribe in primary care. Prim Health Care Res Dev 2008;9:199-204.

33. Malani AN, Brennan BM, Collins CD, et al. Antimicrobial stewardship practices in Michigan long-term care facilities. Infect Control Hosp Epidemiol 2016;37:236-7.

34. Manning ML, Giannuzzi D. Keeping patients safe: antibiotic resistance and the role of nurse executives in antibiotic stewardship. J Nurs Adm 2015;45:67-9.

35. Ness V, Malcolm W, McGivern G, Reilly J. Growth in nurse prescribing of antibiotics: the scottish experience 2007-13. J Antimicrob Chemother 2015;70:3384-9.

36. Maier CB, Aiken LH. Task shifting from physicians to nurses in primary care in 39 countries: a cross-country comparative study. Eur J Public Health 2016;26:927-34.
37. Courtenay M, Carter S, Rowbotham S, Peters S. Antibiotic prescribing in primary care: The need for interprofessional collaboration. J Interprof Care 2015;29:404-5.

38. Scottish Medicines Consortium, Scottish Antimicrobial Prescribing Group. Exploring the role of nurses and midwives in antimicrobial stewardship. 2014. Available from: http://www.nes. scot.nhs.uk/media/3065666/exploring_r ole_of_nurses_and_midwives_in_anti microbial_stewardship_report.pdf. Accessed 24/09/2016

39. Dunn K, O'Reilly A, Silke B, et al. Implementing a pharmacist-led sequential antimicrobial therapy strategy: a controlled before-and-after study. Int J Clin Pharm 2011;33:208-14.

40. Wickens HJ, Farrell S, Ashiru-Oredope $\mathrm{DA}$, et al. The increasing role of pharmacists in antimicrobial stewardship in English hospitals. J Antimicrob Chemother 2013;68:2675-81.

41. Charani E, Kyratsis Y, Lawson W, et al. An analysis of the development and implementation of a smartphone application for the delivery of antimicrobial prescribing policy: lessons learnt. J Antimicrob Chemother 2013;68:960-7.

42. Sneddon J, Gilchrist M, Wickens H. Development of an expert professional curriculum for antimicrobial pharmacists in the UK. J Antimicrob Chemother 2015;70:1277-80.

43. Hidayat L, Patel S, Veltri K. Activelearning implementation in an advanced elective course on infectious diseases. Am J Pharm Educ 2012;76:87.

44. Dar-Odeh NS, Abu-Hammad OA, AlOmiri MK, et al. Antibiotic prescribing practices by dentists: a review. Ther Clin Risk Manag 2010;6:301-6.

45. Laheij AM, Kistler JO, Belibasakis GN, et al. Healthcare-associated viral and bacterial infections in dentistry. J Oral Microbiol 2012;4:17659.

46. Lewis MA. Why we must reduce dental prescription of antibiotics: European Union Antibiotic Awareness Day. Br Dent J 2008;205:537-8.

47. Elouafkaoui P, Young L, Newlands R, et al. An audit and feedback intervention for reducing antibiotic prescribing in general dental practice: The RAPiD cluster randomised controlled trial. PLoS Med 2016;13:e1002115.

48. Royal Pharmaceutical Society. A competency framework for all prescribers. 2016. Available from: http://www. rpharms.com/support-pdfs/prescribingcompetency-framework.pdf. Accessed 24/09/2106

49. Nathwani D, Sneddon J, Malcolm W, et 
al. Scottish Antimicrobial Prescribing Group (SAPG): development and impact of the Scottish National Antimicrobial Stewardship Programme. Int J Antimicrob Agents 2011;38:16-26.

50. Castro-Sánchez ECE, Moore LSP, Gharbi M, Holmes AH. On call: antibiotics - development and evaluation of a serious antimicrobial prescribing game for hospital care. In: Schouten BFS, Schijven M, Vosmeer M, Gekker A, eds. 4th conference on gaming and playful interaction in healthcare. Wiesbaden: Springer Vieweg; 2014. p. 1-8.

51. Pan X, Slater M, Beacco A, et al. The responses of medical general practitioners to unreasonable patient demand for antibiotics - a study of medical ethics using immersive virtual reality. PLoS One 2016;11:e0146837.

52. Gould LM. Minimum antibiotic stewardship measures. Clin Microbiol Infect 2001;7:22-6.

53. Wickens HJ, Farrell S, Ashiru-Oredope DA, et al. The increasing role of pharmacists in antimicrobial stewardship in English hospitals. J Antimicrob Chemother 2013;68:2675-81.

54. Brink AJ, Messina AP, Feldman C, et al. Antimicrobial stewardship across 47 South African hospitals: an implementation study. Lancet Infect Dis 2016;16:1017-25.

55. Gabbay J, le May A. Evidence based guidelines or collectively constructed mindlines? Ethnographic study of knowledge management in primary care. BMJ 2004;329:1013.

56. Ansari F, Gray K, Nathwani D, et al. Outcomes of an intervention to improve hospital antibiotic prescribing: interrupted time series with segmented regression analysis. J Antimicrob Chemother 2003;52:842-8.

57. Thakkar K, Gilchrist M, Dickinson E, et al. A quality improvement programme to increase compliance with an antiinfective prescribing policy. J Antimicrob Chemother 2011;66:191620.

58. Gjelstad S, Hoye S, Straand J, et al. Improving antibiotic prescribing in acute respiratory tract infections: cluster randomised trial from Norwegian general practice (prescription peer academic detailing (Rx-PAD) study). BMJ 2013;347:f4403.

59. Mauffrey V, Kivits J, Pulcini C, Boivin JM. Perception of acceptable antibiotic stewardship strategies in outpatient settings. Med Malad Infect 2016;46:28593.

60. Nand P, Wilson MD, Cohen SH, Brown J. Curbing antimicrobial resistance: do physicians receive adequate education about antibiograms? J Infect 2016;72:127-9.

61. Coyne LA, Pinchbeck GL, Williams NJ, et al. Understanding antimicrobial use and prescribing behaviours by pig veterinary surgeons and farmers: a qualitative study. Vet Record 2014;175:593.

62. Duane S, Domegan C, Callan A, et al. Using qualitative insights to change practice: exploring the culture of antibiotic prescribing and consumption for urinary tract infections. BMJ 2016;6:e008894.

63. Treweek S, Francis JJ, Bonetti D, et al. A primary care web-based intervention modeling experiment replicated behavior changes seen in earlier paper-based experiment. J Clin Epidemiol 2016;80:116-22.

64. Grondal H, Hedin K, Strandberg EL, et al. Near-patient tests and the clinical gaze in decision-making of Swedish GPs not following current guidelines for sore throat: a qualitative interview study. BMC Fam Pract 2015;16:81.

65. Grayson ML, Macesic N, Huang GK, et al. Use of an innovative personalitymindset profiling tool to guide culturechange strategies among different healthcare worker groups. PLoS One 2015;10:e140509.

66. Ashiru-Oredope D, Sharland M, Charani E, et al. Improving the quality of antibiotic prescribing in the NHS by developing a new antimicrobial stewardship programme: start smart-then focus. J Antimicrob Chemother 2012;67:i51-63.

67. Rawson TM, Moore LS, Gilchrist MJ, Holmes AH. Antimicrobial steward- ship: are we failing in cross-specialty clinical engagement? J Antimicrob Chemother 2016;71:554-9.

68. Cosgrove SE, Hermsen ED, Rybak MJ, et al. Guidance for the knowledge and skills required for antimicrobial stewardship leaders. Infect Control Hosp Epidemiol 2014;35:1444-51.

69. Rocha BH, Christenson JC, Evans RS, Gardner RM. Clinicians' response to computerized detection of infections. JAMIA 2001;8:117-25.

70. Baysari MT, Lehnbom EC, Li L, et al. The effectiveness of information technology to improve antimicrobial prescribing in hospitals: a systematic review and meta-analysis. Int $\mathrm{J}$ Med Inform 2016;92:15-34.

71. Zingg W, Holmes A, Dettenkofer M, et al. Hospital organisation, management, and structure for prevention of healthcare-associated infection: a systematic review and expert consensus. Lancet Infect Dis 2015;15:212-24.

72. Oppenheimer M, Rezwan N. CQUIN audit for prescription of antibiotics for urinary tract infections in an acute medical assessment unit. BMJ Qual Improv Rep 2015;4:u208446.w3374.

73. van den Bosch CM, Geerlings SE, Natsch S, et al. Quality indicators to measure appropriate antibiotic use in hospitalized adults. Clin Infect Dis 2015;60:281-91.

74. Minejima E, Wong-Beringer A. Implementation of rapid diagnostics with antimicrobial stewardship. Exp Rev Anti Infect Ther 2016:1-11.

75. Jones CH, Howick J, Roberts NW, et al. Primary care clinicians' attitudes towards point-of-care blood testing: a systematic review of qualitative studies. BMC Fam Pract 2013;14:117.

76. Moore LS, Freeman R, Gilchrist MJ, et al. Homogeneity of antimicrobial policy, yet heterogeneity of antimicrobial resistance: antimicrobial non-susceptibility among 108,717 clinical isolates from primary, secondary and tertiary care patients in London. J Antimicrob Chemother 2014;69:3409-22. 